\title{
Conceptualizing and Measuring How Employees and Organizations Manage Uncertainty
}

\author{
Phillip G. Clampitt \\ Professor at University of Wisconsin - Green Bay \\ MAC C321 UWGB \\ Green Bay, WI 54311 \\ Phone: 920.465.2324 Fax: 920.465.2890 \\ E-mail: Clampitt@imetacomm.com \\ M. Lee Williams \\ Professor at Texas State University-San Marcos \\ Department of Communication Studies \\ San Marcos, TX 78666 \\ Phone: 512.245.2165 Fax: 512.245.3138 \\ E-mail:mw02@txstate.edu
}

\begin{abstract}
The study of uncertainty is central to an understanding of organizational communication. This research investigates the organizational work climates that emerge from the way employees manage uncertainty as well as the way they perceive their organization managing uncertainty. The Uncertainty Management Matrix is presented as a framework for conceptualizing this construct, followed by three scale development studies. The article concludes with a variety of research questions to direct future communication research.
\end{abstract}


The academic study of “uncertainty” has enjoyed a long and wide-ranging history, and theorists have conceived this rich construct from a variety of perspectives. In physics, uncertainty is seen as the inherent state of nature (Atkins, 1984). In information theory, Claude Shannon envisions uncertainty as a state of disorder (i.e., noise) that must be overcome so that a signal can be transmitted from point A to point B (Pierce, 1961; Shannon \& Weaver, 1949). In personality trait research, uncertainty is investigated as a person’s predisposition to tolerate ambiguity (Budner, 1962; Norton, 1975). Those with greater intolerance for ambiguity tend to resort to black-white solutions, exhibit premature closure, and often ignore reality (Frenkel-Brunswik, 1949). In interpersonal communication, uncertainty reduction theory postulates that during initial encounters people naturally experience uncertainty and seek to reduce it by gathering more information (Berger \& Calabrese, 1975). In the study of cultures, Hofstede (1980) created the Uncertainty Avoidance Index to assess the degree to which certain cultures dispose individuals to avoid or embrace uncertainty. In health communication, uncertainty management theory asserts that individuals do not always seek to reduce uncertainty but sometimes (especially in the presence of negative information) reject clarity, seeking ambiguity and even confusion (Babrow, Hines, \& Kasch, 2000; Bradac, 2001; Brashers, Neidig, Haas, Dobbs, Cardillo, \& Russell, 2000).

\section{UNCERTAINTY, COMMUNICATION, AND ORGANIZATIONS}

Understanding uncertainty has also been an area of major interest in organizations, and this is the emphasis of the study reported here. Organizational uncertainty research has concentrated on two primary dimensions - individual employee uncertainty and uncertainty in the external environment. Employees experience uncertainty when there is a difference between information available and information needed (Goldhaber, 1993). If information is needed but if it is limited, ambiguous, complex, unpredictable, or probabilistic, than the result is uncertainty. Therefore, if an employee is in a state of uncertainty, a symbolic transmission can provide a discernable pattern (i.e., 
information), and if the participants share a common symbol system, than some degree of communication can occur (Farace, Monge, \& Russell, 1977). In addition, Brashers (2001) identifies a variety of types of internal uncertainty that must be managed in organizational life. Newcomers must cope with the uncertainties of how to do their job, expectations for acceptable performance, relationships with others, organizational values, and organizational politics. More seasoned employees must deal with the uncertainties of role ambiguity, job transfers, organizational change, and implementing innovations.

Weick (1979; 1995) identifies uncertainty as a major factor in organizations when he contends that it is inevitable and the basis for organizational sensemaking. Employees do not simply react to messages, but through the process of enactment they select from many interpretations to configure and retain meanings. Still another perception of uncertainty is that of Eisenberg (1984), who argues that it is not necessarily bad and can provide a variety of benefits. He asserts that ambiguity can be used strategically to create cohesiveness when there is disagreement between parties as well as flexibility in policies and procedures, thus expediting change.

The uncertainty of external environments has also been a long-standing area of concern for organizational researchers (Emery \& Trist, 1965; Katz \& Kahn, 1978). The stability of markets, availability of resources, competitors, customer demands, and changing economic conditions can greatly impact the complexity, and thus uncertainty, of organizational decision-making. According to the law of requisite variety (Ashby, 1956), organizational structures and communication must match the complexity of the external environment if an organization hopes to survive. As circumstances become more uncertain, organizations must become more complex in an effort to adapt. Chaotic environments are much more of the norm for contemporary organizations, and analytical techniques such as strategic planning and cost-benefit analysis are designed to categorize, quantify, and reify the future (Clampitt \& DeKoch, 2001). These processes have been developed to 
understand the level of environmental uncertainty so as to create a strategic posture to maximize effectiveness (Courtney, Kirkland, and Viguerie, 1997).

In sum, organizational and organizational communication literature suggests that how employees and organizations manage uncertainty is a fundamental concern of the field. Yet, surprisingly little work has been done to conceptualize and measure these seemingly intertwined issues.

\section{CONCEPTUALIZING UNCERTAINTY MANAGEMENT IN ORGANIZATIONS}

As discussed in the previous section, individuals vary in the way they approach and deal with uncertainty. While some embrace it, many are uncomfortable with uncertainty due to the inherent lack of predictability. It tends to be cognitively and emotionally challenging. It creates a feeling of vulnerability or anxiety that can lead to actively distorting perceptions and information. This can produce false dichotomies, resistance to change, rejection of relevant information, rigid categories, and regression to old rule-of-thumb models of thinking (Clampitt \& DeKoch, 2001).

Just as individuals must deal with the challenges of uncertainty, so must organizations. Some organizations manage uncertainty by embracing it, openly discussing changes in their customer base and competitors, fostering innovation, encouraging meaningful dialogue, and deemphasizing rigid planning processes. Others, however, tend to avoid uncertainty by following inflexible control procedures or policies, ignoring changing circumstances, overly relying on success recipes, and artificially bolstering organizational successes at the expense of overlooking shortcomings.

While organizational research has addressed individual uncertainty management as well as how the organization manages external uncertainty, these separate constructs have not been investigated jointly from the employee's perspective. It is our belief that this is an unfortunate oversight that limits an understanding of the context in which employees make decisions about their 
communication and behavior. Therefore, the objective of the current investigation is to extend the uncertainty construct and to conceptualize it not only as an individual variable but one that is moderated by how employees perceive the organization addressing uncertainty and change in the external environment. The assumption is that the integration of these separate dimensions of uncertainty will create a more meaningful construct, and thus provide greater insight into organizational communication.

This new conceptualization of uncertainty management is displayed in the Uncertainty Management Matrix (see Figure 1). This framework juxtaposes the individual employee’s approach to uncertainty and how s/he perceives the organization's approach to uncertainty. Those individuals that embrace uncertainty see it as challenging, desirable, invigorating, and useful. They do not try to artificially drive the ambiguities and contradictions out of the situation. Conversely, those individuals that avoid uncertainty view it as threatening and undesirable. They tend to shun complexities and novelty, and prematurely structure ambiguous situations. In like manner, individuals can perceive their organization as embracing uncertainty (i.e., being open to change and innovative) or see their organization as avoiding uncertainty by denying the presence or need for change.

Depending on how the individual employee approaches uncertainty and the way s/he perceives how the organization embraces or avoids uncertainty, different climates emerge. Each quadrant represents a different kind of organizational climate, with varying beliefs, values, assumptions, and ways of communicating. As suggested in Figure 1, there are four basic possibilities:

- Status Quo Climate: Employees and the organization both avoid uncertainty. Employees want few surprises and they rarely get them.

- Unsettling Climate: Employees desire certainty while they perceive the organization as embracing uncertainty. Thus employees become unsettled and perhaps overwhelmed by the chaotic work environment. 
- Stifling Climate: Employees embrace uncertainty but they perceive that the organization avoids it. The result is that employees feel stifled.

- Dynamic Climate: Both employees and the organization embrace uncertainty. Employees want change and progress, and the organization promotes it.

The framework conceptualized in the Uncertainty Management Matrix is viewed as valuable for three primary reasons. First, it identifies uncertainty as a central variable individuals and organizations must manage so as to compete and survive in an increasingly complex world (Berger, 1987; Berger and Calabrese, 1975; Goldhaber, 1993). To be successful in the information era, organizations must understand the processing inclinations and capacities of individual employees as well as the organization, and create messages that are appropriate for different audiences (Clampitt \& Williams, 2004). Second, the model is based on the assumption that the organizational work environment is best viewed as an individual perception and not an ontological or self-evident reality. While an organization might project espoused or idealized qualities, the way employees perceive the organization managing uncertainty is viewed as a more useful approach to understanding organizational behavior (Schein, 1992). Employees are active information processors who act on their perceptions. Third, this framework seeks to integrate an individual difference variable with a sociological variable. While personality can influence behavior, rarely is one factor so powerful that it dominates in every social setting (Infante, Rancer, \& Womack, 2003). The interaction of how an employee approaches uncertainty and the perception of how the organization manages uncertainty should provide a better model for understanding organizational behavior than looking at either variable individually.

In an effort to operationalize uncertainty management, three separate studies were undertaken to develop and refine the scales. What follows is a description of these studies. 


\section{STUDY 1: INITIAL SCALE DEVELOPMENT}

The purpose of this initial analysis was to operationalize: (1) how employees manage their personal uncertainty, and (2) how they see their organization managing uncertainty. An additional objective was to delineate the factor structure of each of these constructs.

We first generated a pool of 45 declarative statements designed to measure how employees manage personal uncertainty. These addressed topics such as acknowledging the existence of uncertainty, processing uncertainty, communicating about uncertainty, and appropriately responding to uncertainty. Using a Likert scale, both positive and negative statements were produced. In an effort to measure how employees perceived their organization managing uncertainty (i.e., work environment uncertainty), a separate pool of 46 items was created.

Participants in the preliminary study were drawn from a wide range of organizations in Wisconsin, Florida and Texas. The size of the organizations ranged from 10 employees to 10,000. Questionnaires were distributed to a cross section of 301 managerial and non-managerial employees. A total of 200 usable questionnaires were returned. Sixty percent of the respondents were female and $40 \%$ male. The age of employees ranged from 19 to 82 years $(M=39.7, S D=13.2)$.

We used principal components factor analysis with orthogonal (varimax) rotation to analyze the responses to the personal uncertainty items and work environment uncertainty items. Guidelines prescribed by McCroskey and Young (1979) were used to select factors. Items were eliminated or considered for rewording depending on their factor loading, item-whole correlation, contribution to the reliability of the factor, and the number of items needed to create a reliable factor.

The factor analysis of the 45 personal uncertainty items produced 9 factors with eigenvalues above 1.0. However, most of the factors had low weightings or contained less than three items loading on the factor. After evaluating the results, items were deleted and subsequent factor 
analyses were conducted. The optimal solution appeared to be one that included 16 items clustered into 4 factors explaining $55.5 \%$ of the variance.

The initial factor analysis of the 46 items on how the organization manages uncertainty produced 12 factors with eigenvalues greater than 1.0. Since most of the items were not loaded highly on a factor or an insufficient number of items loaded on a factor, items were eliminated, and additional factor analyses were conducted. Guided by the conceptual framework established for this construct, it was determined that the best solution included 13 items representing 4 factors, which explained $60.0 \%$ of the variance.

\section{STUDY 2: CONTINUED SCALE DEVELOPMENT AND VALIDATION}

The objective of Study 2 was to create scales with a clear factor structure possessing sound reliability and validity. After analyzing the results of the first study, items on the personal uncertainty management measure and work environment uncertainty measure were reworded and new items created. The version of the personal uncertainty management scale used in Study 2 contained 25 items, and the work environment uncertainty measure contained 22 items.

The questionnaire employed in Study 2 consisted of 82 total items. It was posed as a survey of the work climate, and it included 25 personal uncertainty management items, 22 work environment uncertainty items, the 10-item Social Desirability Scale (Strahan \& Gerbask, 1972), the 16-item Intolerance of Ambiguity Scale (Budner, 1962), five demographic items, and four outcome measures related to job satisfaction, commitment to the organization, and general comfort with uncertainty.

Subjects used in this phase of the research were drawn from a broad spectrum of organizations including state government agencies, a paper company, financial institutions, health organizations, universities, manufacturing and retail organizations. A total of 239 managerial and non-managerial employees completed the questionnaire. Sixty-one percent were female and 39\% 
were male. The age of employees ranged from 19 to 67 years $(\mathrm{M}=41.0, \mathrm{SD}=11.0)$, and job tenure ranged from 1 month to 40 years $(\mathrm{M}=8.7$ years, $\mathrm{SD}=8.9)$.

Items from the personal uncertainty management measure were submitted to principal components factor analysis with orthogonal (varimax) rotation using the 60/40 criterion (McCroskey \& Young, 1979). The analysis produced seven factors with eigenvalues greater than 1.0. An examination of the factor loadings and scree plot revealed that a three-factor interpretation (accounting for $55.6 \%$ of the variance) was appropriate. The four items on the first factor addressed process uncertainty and the employee's comfort in making a decision on intuition or a hunch. The four items on the second factor addressed the employee's need to have detailed plans or know the specific outcome of a task or project. The three items on the third factor addressed the individual's willingness to perceive uncertainty as well as to actively look at different perspectives, new ideas, or signs that the situation is changing. Cronbach's alpha reliability values for each of the factors were $.74, .70$, and .67 , respectively, and the overall alpha reliability was .70 . The composite score for the 11-item personal uncertainty management scale produced a mean of 52.0, median of 52, and standard deviation of 8.28 .

Similar procedures were used with the work environment uncertainty management items. The factor analysis produced five factors with eigenvalues greater than 1.0. An examination of the factor loadings and scree plot revealed that a three-factor interpretation (accounting for $62.1 \%$ of the variance) was appropriate. The four items on the first factor addressed the degree to which the organization encouraged employees to express doubts or misgivings. The four items on the second factor addressed the degree to which the organization was willing to actively look for new ideas to address problems or signs that the situation was changing. The three items on the third factor addressed the degree to which the organization needed detailed plans or a specific outcome before starting a project. Cronbach’s alpha reliability values for each of the factors were .78, .77, and .70, 
respectively, and the overall alpha reliability was .78. The composite score for the 11-item work environment uncertainty management scale produced a mean of 47.9 , median of 49 , and standard deviation of 10.0 .

To determine the convergent and divergent validity of the personal uncertainty management scale, it was correlated with a variety of conceptually related scales. As anticipated, personal uncertainty management was significantly correlated with Budner's (1962) Intolerance of Ambiguity Scale $(r=-.40, \mathrm{p}<.001)$. This concurrent validity finding indicates that an employee who does not embrace uncertainty is also intolerant of ambiguous situations. The personal uncertainty management scale was also correlated with a single item measuring comfort with uncertainty $(\mathrm{r}=$ $.39, \mathrm{p}<.001)$, but not significantly correlated with gender $(r=-.07)$, age $(r=-.05)$, or tenure in the organization $(r=.05)$. The scale was significantly correlated with managerial level $(r=-.23, p<.001)$, thus indicating employees in non-managerial positions do not embrace uncertainty as much as employees in managerial positions. Personal uncertainty management was not correlated with the Social Desirability Scale (Strahan \& Gerbask, 1972) ( $\mathrm{r}=.05)$, nor was it correlated with a single item measure of job satisfaction $(r=.10)$ or commitment $(r=.12)$.

To assess the validity of the work environment uncertainty management scale, it was also correlated with a variety of other scales. Work environment uncertainty was not correlated with Intolerance of Ambiguity (Budner, 1962) $(r=-.02)$, thus indicating employees’ personal tolerance of ambiguity was not related to how they perceived their organization managing uncertainty. In addition, the work environment uncertainty management scale was not correlated with the individual's comfort with uncertainty $(r=-.02)$, gender $(r=-.02)$, age $(r=.01)$, tenure in the organization $(r=.07)$, or managerial level $(r=-.07)$. Results also revealed that work environment uncertainty management was minimally correlated with Social Desirability (Strahan \& Gerbask, 1972) $(r=.15, \mathrm{p}<.02)$. Since this correlation explained only $2 \%$ of the variance, this relationship was 
considered inconsequential. Finally, the work environment uncertainty management scale was not significantly correlated with the personal uncertainty management scale $(r=.06)$. Overall, these results for the two scales are reassuring. The scales were not conceptually redundant with any of the other variables investigated in the study, and the scales did not evoke socially desirable responses. The scales are not correlated with each other, and they tap conceptually distinctive dimensions of uncertainty management.

\section{STUDY 3: SCALE REFINEMENT}

The objective of the third study was to build on the findings of Study 2, generate several new items so that each factor in the scales contained 4 items, confirm the factor structure, create a large database of employees from diverse organizations, and establish norms for the scales. Organizational liaisons distributed questionnaires to a cross section of managerial and nonmanagerial employees. Respondents anonymously mailed their surveys to the researchers in selfaddressed stamped envelopes or returned them to the organizational liaison.

A total of 1,046 questionnaires were completed and analyzed over a three-year time period. The large majority of subjects came from across the United States, with a few from Canada. Included were $62.9 \%$ females and $37.1 \%$ males. The age of employees ranged from 16 to 74 years $(\mathrm{M}=39.8, \mathrm{SD}=11.2)$, and job tenure ranged from 1 month to 45 years $(\mathrm{M}=7.0$ years, $\mathrm{SD}=9.0)$. The distribution of subjects by managerial level was $10.0 \%$ top management, 38.3\% management, 27.0\% non-managerial professional, $19.8 \%$ non-management, and $4.9 \%$ other. The organizations included in the sample were 39.2\% non-profit (e.g., education, government), $18.3 \%$ service-oriented (e.g., health care, retail, sales, marketing, hospitality), 17.5\% industrial (e.g., manufacturing, construction, utilities, transportation), 13.9\% financial (e.g., insurance, banks, financial institutions), 9.2\% information technology (e.g., media/communications, technology, research, publishing), and $2 \%$ other. 
Results for the Personal Uncertainty Management (PUM) Scale

The result of the factor analysis produced a three-factor solution accounting for $58.4 \%$ of the total variance. This final version of the scale is presented in Table 1. Eigenvalues were 2.91, 2.46, and 1.64, respectively. The first factor accounted for $24.2 \%$ of the variance and was named "Process Uncertainty.” The four items on this factor addressed the employee’s comfort in making a decision on intuition or a hunch. The second factor, which accounted for $20.5 \%$ of the variance, contained four items and was named “Outcome Uncertainty.” These items addressed the employee's need to have detailed plans or know the specific outcome of a task or project. The third factor, which accounted for $13.7 \%$ of the variance, was named "Perceptual Uncertainty.” The four items on this factor addressed the individual's willingness to actively look at different perspectives, new ideas, or signs that the situation is changing.

In this study, personal uncertainty management is treated as a single, general construct even though it has underlying factors. For this reason, the composite sum score is used instead of individual factors. This procedure is commonly accepted when researchers want to operationalize a construct in its broadest sense (Daily, Vangelista, \& Daughton, 1987; Fry, Botan, \& Kreps, 2000; Levine \& McCroskey, 1990) or when the composite score is more strongly related to other variables than the underlying factors (Snyder \& Gangestad, 1986). The composite sum score for the PUM Scale produced a mean of 57.19, median of 57 , and standard deviation of $8.36(n=1046)$. A visual inspection of the frequency distribution revealed the scale was normally distributed. The overall Cronbach’s alpha reliability was .69, and alpha reliability values for each of the factors were .72, .77 , and .76, respectively. Results for the Work Environment Uncertainty Management (WEUM) Scale

The result of the factor analysis for work environment uncertainty management produced a three-factor solution accounting for $60.5 \%$ of the total variance. The final version of the scale is 
presented in Table 2. Eigenvalues were 3.39, 2.55, and 1.32, respectively. The first factor accounted for $28.3 \%$ of the variance and was named "Expressed Uncertainty.” The four items on this factor addressed the degree to which the organization encouraged employees to express doubts or misgivings. The second factor, which accounted for $21.2 \%$ of the variance, contained four items and was named “Outcome Uncertainty.” These items addressed the degree to which the organization needed detailed plans or a specific outcome before starting a project. The third factor, which accounted for $11.0 \%$ of the variance, was named "Perceptual Uncertainty." The four items on this factor addressed the degree to which the organization was willing to actively look for new ideas to address problems or signs that the situation was changing.

The composite sum score for the WEUM Scale produced a mean of 51.43, median of 51, and standard deviation of $9.67(\mathrm{n}=1046)$. Scores were normally distributed. The overall Cronbach’s alpha reliability was .73, and alpha reliability values for each of the factors were .73, .80 , and .76, respectively.

\section{PLOTTING SCORES ON THE UNCERTAINTY MANAGEMENT MATRIX}

The Uncertainty Management Matrix was conceptualized and described earlier (see Figure 1). Using the scales developed to measure how employees manage their personal uncertainty and how they perceive their organization managing uncertainty, we can now operationalize each of the four uncertainty management climates. (Note: For a copy of the scales and scoring procedures, consult the following website: $\underline{\text { www.imetacomm.com })}$

The 12 items on the PUM Scale have a potential range from 12 to 84 with a median score of 57. The 12 items on the WEUM Scale have a potential range from 12 to 84 with a median score of 51. By employing a median split, respondents were divided into high or low categories on each scale. It was then possible to place a subject into one of the four quadrants of the Uncertainty Management Matrix. After plotting the scores of respondents in the database, the results indicated that $28.5 \%$ 
were in the Status Quo Climate ( $n=298), 22.2 \%$ were in the Unsettling Climate $(n=232), 22.6 \%$ were in the Stifling Climate ( $n=236)$, and $26.8 \%$ were in the Dynamic Climate $(n=280)$.

\section{FUTURE RESEARCH}

The Uncertainty Management Matrix and instruments presented in this study can provide a useful line of inquiry in organizations. By using this framework, a variety of general research questions can be considered for future research:

- Which climates (i.e., Status Quo, Unsettling, Stifling, Dynamic) exhibit the highest satisfaction, organizational commitment, identification with the organization, and productivity?

- What beliefs and value systems dominate in the various climates?

- Are demographics such as gender, age, and tenure equally distributed across the various climates, or do certain demographics cluster in different climates?

- Are different types of organizations (e.g., non-profit, service, industrial, financial) more oriented toward certain climates?

- What are the "best fit" implications for new employees? Should individuals who avoid uncertainty seek employment in a Status Quo climate, and should individuals who embrace uncertainty seek out a Dynamic climate?

In addition, research questions directed more toward communication would include the following:

- Which climates exhibit the highest degree of satisfaction with organizational communication or communication with the immediate supervisor?

- What types of storytelling take place in each climate? Are the sense-making processes and outcomes different in the various climates?

- Is organizational dissent more prevalent in certain climates, and if so, which types of dissent dominate?

- What manager communication strategies are most effective when interacting with employees in each climate? Are managers more likely to use certain compliance-gaining strategies in different climates?

- What communication strategies are most effective when change is desired? For example, how can a Status Quo climate be changed to an Unsettling climate, and then to a Dynamic climate? What strategies can employees use in a Stifling climate to direct the organization more toward a Dynamic climate?

In sum, the scales developed to measure the climates in the Uncertainty Management Matrix are psychometrically sound and practical. They are internally reliable and exhibit convergent as well as divergent validity. Most respondents can complete the scales in less than 10 minutes, and scoring is 
straightforward. Furthermore, this framework can provide researchers and practitioners with an insightful perspective for understanding communication practices associated with managing the inherent uncertainty that pervades organizations.

\section{REFERENCES}

Ashby, W.R. (1956). An introduction to cybernetics. London: Chapman \& Hall.

Atkins, P.W. (1984). The second law. New York: Scientific American Books.

Babrow, A. S., Hines, S. C., \& Kasch, C. R. (2000). Managing uncertainty in illness explanations: An application of problematic integration theory. In B. Whaley (Ed.), Explaining illness: Research, theory, and strategies (pp. 41-67). Hillsdale, NJ: Erlbaum.

Berger, C. R. (1987). Communicating uncertainty. In M. E. Roloff \& G. R. Miller (Eds.), Interpersonal processes: New directions in communication research (pp. 39-62). Beverly Hills, CA: Sage.

Berger, C. R, \& Calabrese, R. J. (1975). Some explorations in initial interaction and beyond: Toward a developmental theory of interpersonal communication. Human Communication Research, 1, 99-112.

Bradac, J. J. (2001). Theory comparison: Uncertainty reduction, problematic integration, uncertainty management, and other curious constructs. Journal of Communication, 51, 456476.

Brashers, D. E. (2001). Communication and uncertainty management. Journal of Communication, $51,477-497$.

Brashers, D. E., Neidig, J. L., Haas, S. M., Dobbs, L. K., Cardillo, L. W., \& Russell, J. A. (2000). Communication in the management of uncertainty: The case of persons living with HIV or AIDS. Communication Monographs, 67, 63-84. 
Budner, S. (1962). Intolerance of ambiguity as a personality variable. Journal of Personality, 30, 29-59

Clampitt, P., \& DeKoch, B. (2001). Embracing uncertainty: The essence of leadership. New York: M.E. Sharpe.

Clampitt, P., \& Williams, M. L. (2004). Communicating about organizational uncertainty. In D. Tourish \& O. Hargie (Eds.), Key issues in organizational communication (pp. 37-59). New York: Routledge.

Courtney, H., Kirkland, J., \& Viguerie, P. (1997). Strategy under uncertainty. Harvard Business Review, 75(6), 66-81.

Daily, J. A., Vangelista, A. L., \& Daughton, S. M. (1987). The nature and correlates of conversational sensitivity. Human communication research, 14, 167-202.

Eisenberg, E. M. (1984). Ambiguity as a strategy in organizational communication. Communication Monographs, 51, 227-242.

Emery, F., \& Trist. E. L. (1965). The causal texture of organizational environments, Human Relations, 18, 21-32.

Farace, R. V., Monge, P. R., \& Russell, H. M. (1977). Communicating and organizing. Reading, MA: Addison-Wesley.

Frenkel-Brunswik, E. (1949). Intolerance of ambiguity as an emotional perceptual personality variable. Journal of Personality, 18, 108-143.

Fry, L. R., Botan, C. H., \& Kreps, G. L. (2000). Investigating communication: An introduction to research methods ( $2^{\text {nd }}$ ed.). Boston: Allyn and Bacon.

Goldhaber, G. M. (1993). Organizational communication ( $6^{\text {th }}$ ed.). Dubuque, IA: William C. Brown and Benchmark . 
Hofstede, G. (1984). Culture’s consequences: International differences in work-related values. Beverly Hills, CA: Sage Publications.

Infante, D. A., Rancer, A. S., \& Womack, D. F. (2003). Building communication theory (4 ${ }^{\text {th }}$ ed.). Prospect Heights, IL: Waveland Press.

Katz, D., \& Kahn, R. (1978). The social psychology of organizations (2 ${ }^{\text {nd }}$ ed.). New York: Wiley. Levine, T. R., \& McCroskey, J. C. (1990). Measuring trait communication apprehension: A test of rival measurement models of the PRCA-24. Communication Monographs, 57, 2-72.

McCroskey, J. C., \& Young, T. J. (1979). The use and abuse of factor analysis in communication research. Human Communication Research, 5, 375-382.

Norton, R. W. (1975). Measurement of ambiguity tolerance. Journal of Personality Assessment, 39, 607-619.

Pierce, J. R. (1961). Symbols, signals, and noise: The nature and process of communication. New York: Harper \& Row.

Schein, E. H. (1992). Organizational culture and leadership ( $2^{\text {nd }}$ ed.). San Francisco, CA: JosseyBass.

Shannon, C., \& Weaver, W. (1949). The mathematical theory of communication. Urbana, IL: University of Illinois Press.

Snyder, M., \& Gangestad, S. (1986). On the nature of self-monitoring: Matters of assessment, matters of validity. Journal of Personality and Social Psychology, 51, 125-139.

Strahan, R., \& Gerbasi, K. (1972). Short, homogenous version of the Marlowe-Crowne Social Desirability Scale, Journal of Clinical Psychology, 28, 191-193.

Weick, K .E. (1995). Sensemaking in organizations. Thousand Oaks, CA: Sage Publications. Weick, K. E. (1979). The social psychology of organizing ( $2^{\text {nd }}$ ed.). Reading, MA: Addison-Wesley. 
FIGURE 1

The Uncertainty Management Matrix

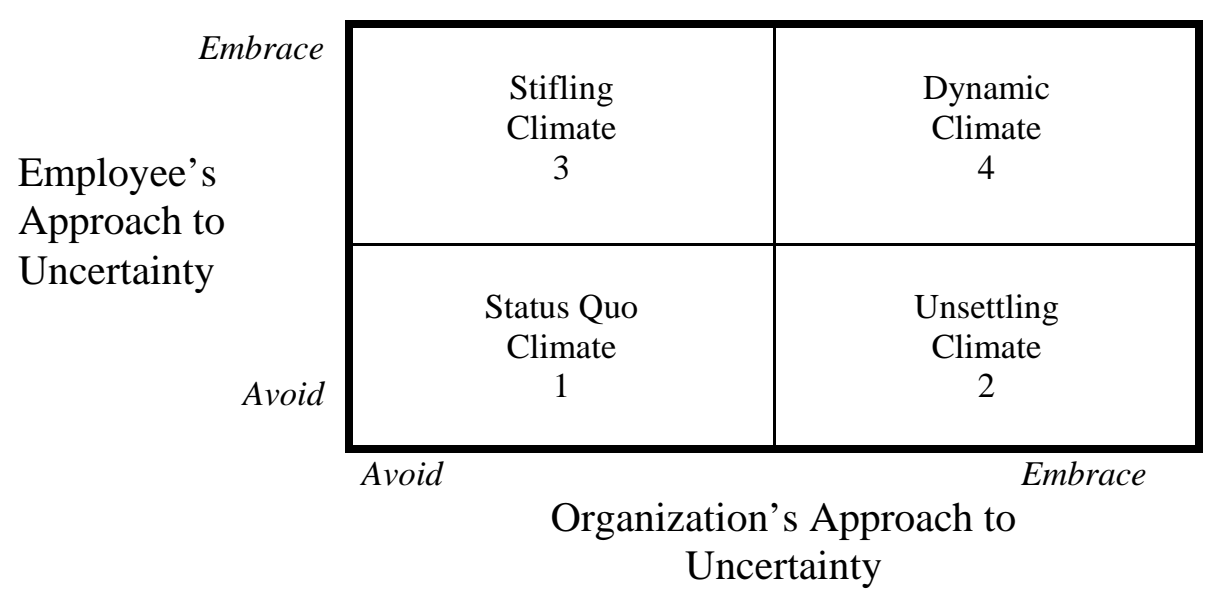

TABLE 1

Personal Uncertainty Management (PUM) Scale Items and Factor Loadings

\begin{tabular}{cccc}
\hline Item & $\begin{array}{c}\text { Factor 1 } \\
\text { (Process) }\end{array}$ & $\begin{array}{c}\text { Factor 2 } \\
\text { (Outcome) }\end{array}$ & $\begin{array}{c}\text { Factor 3 } \\
\text { (Perceptual) }\end{array}$ \\
\hline
\end{tabular}

9. I'm willing to make a decision based on a hunch. $\quad .79$

5. I'm comfortable using my intuition to make a decision. $\quad .79$

1. I'm comfortable making a decision on my gut instincts.

14. I'm comfortable deciding on the spur-of-the-moment.

7. I need to know the specific outcome before starting a task. (-) 85

4. When I start a project, I need to know exactly where I'll end up. (-)

13. I need a definite sense of direction for a project (-) $\quad .72$

11. I don’t need a detailed plan when working on a project. $\quad .61$

2. I actively look for signs that the situation is changing.

10. I easily spot changing trends. $\quad .75$

8. I'm quick to notice when circumstances change. $\quad .73$

6. I'm always on the lookout for new ideas to address problems. $\quad .70$ 
TABLE 2

Work Environment Uncertainty Management (WEUM) Scale Items and Factor Loadings

\begin{tabular}{cccc}
\hline Item & $\begin{array}{c}\text { Factor 1 } \\
\text { (Expressed) }\end{array}$ & $\begin{array}{c}\text { Factor 2 } \\
\text { (Outcome) }\end{array}$ & $\begin{array}{c}\text { Factor 3 } \\
\text { (Perceptual) }\end{array}$ \\
\hline
\end{tabular}

33. My organization doesn't want employees to admit that they are unsure about something. (-)

35. My organization discourages employees from talking about their misgivings. (-)

30. My organization doesn't encourage employees to discuss their doubts about a project. (-)

25. In my organization, being unsure about something is a sign of weakness. (-)

31. When my organization starts a project, it needs to know exactly where the project will end up. (-)

29. My organization needs to know the specific outcome before starting a project. (-)

34. My organization wants precise plans before starting a job or project. (-)

27. My organization doesn't need a detailed plan when working on a project.

32. My organization actively looks for signs that the situation is changing.

26. My organization easily spots changing trends.

23. My organization is always on the lookout for new ideas to address problems.

28. Even after my organization makes a decision, it will reevaluate the decision when the situation changes. 